\title{
A Neumann problem for a convection-diffusion equation on the half-line
}

\author{
by Piotr Biler and Grzegorz Karch (Wrocław) \\ In memory of Bogdan Ziemian
}

\begin{abstract}
We study solutions to a nonlinear parabolic convection-diffusion equation on the half-line with the Neumann condition at $x=0$. The analysis is based on the properties of self-similar solutions to that problem.
\end{abstract}

1. Introduction. Our goal in this paper is to study some positive solutions to the initial-boundary value Neumann problem for the convectiondiffusion equation

$$
\begin{aligned}
u_{t}-u_{x x}+\left(u|u|^{q-1}\right)_{x} & =0, \quad x>0, t>0, \\
u_{x}(0, t) & =0 \\
u(x, 0) & =u_{0}(x) .
\end{aligned}
$$

Here $u=u(x, t)$ is a real-valued function of $x \geq 0$ and $t \geq 0$, and $q>1$ is a constant.

Recently, there appeared papers $[4,13,2,12]$ studying properties of solutions of a related equation, where, however, the nonlinear term has a minus sign:

$$
u_{t}-u_{x x}-\left(u|u|^{q-1}\right)_{x}=0
$$

In the first of them, by Claudi et al. [4], the initial data $u_{0}$ is nonnegative, bounded, sufficiently regular, and satisfies $\lim _{x \rightarrow \infty} x^{\alpha} u_{0}(x)=A$ for some $A>0$ and $\alpha>0$ (some restrictions on $\left(u_{0}\right)_{x}$ are also imposed: e.g. $\left(u_{0}\right)_{x}$ $\leq 0)$. Under these assumptions, it is proved that for $0<\alpha<1 /(q-1)$ the long-time behavior of solutions to (1.2)-(1.4) is described by the solution to the conservation law $v_{t}-\left(v^{q}\right)_{x}=0$ for $x>0$ with $v(x, 0)=A x^{-\alpha}$.

2000 Mathematics Subject Classification: 35K15, 35B40.

Key words and phrases: convection-diffusion equation, Neumann problem, asymptotics of solutions. 
The next paper by Peletier and Serafini [13] is devoted to the study of the self-similar solutions to (1.4). In that case, the problem is reduced to an ordinary differential equation of the second order with conditions at 0 and at infinity. The analysis in [13] is based on the so-called "shooting technique" and gives conditions on existence/nonexistence of self-similar profiles for (1.4).

The work by Claudi [2] continues the research began in [4], so that the initial data are assumed to satisfy conditions similar to those in [4]. First, for $q>2$ and $1 /(q-1)<\alpha<1$ Claudi shows in [2, Thm. 1.1] that

$$
t^{\alpha / 2}|u(x, t)-w(x, t)| \rightarrow 0 \quad \text { as } t \rightarrow \infty
$$

uniformly in $x \in \mathbb{R}^{+}$. Here $w(x, t)$ is the self-similar solution to the heat equation $w_{t}-w_{x x}=0$ on the real line with the initial condition $w(x, 0)=$ $A|x|^{-\alpha}$. Next, it is proved in [2, Thm. 1.2] that for $q>2$ and $\alpha>1$ the long-time behavior in $L^{\infty}\left(\mathbb{R}^{+}\right)$of solutions to (1.2)-(1.4) is described by $c_{0}(4 \pi t)^{-1 / 2} \exp \left(-x^{2} /(4 t)\right)$ where $c_{0}=2 \int_{\mathbb{R}^{+}} u_{0}(x) d x-2 \int_{0}^{\infty} u(0, \tau)^{q} d \tau$, namely

$$
t^{1 / 2}\left|u(x, t)-c_{0}(4 \pi t)^{-1 / 2} \exp \left(-x^{2} /(4 t)\right)\right| \rightarrow 0 \quad \text { as } t \rightarrow \infty
$$

uniformly in $x \in \mathbb{R}^{+}$. The paper [2] also deals with the case $1<q<2$ and $\alpha \geq 1 /(q-1)$, and some nonlinear effects are observed in the large-time behavior of solutions.

In the recent work [12], the theory from [2] concerning the asymptotic behavior of solutions to (1.2)-(1.4) with $q>2$ was developed. There, $u_{0}$ is either integrable on $\mathbb{R}^{+}$or behaves like $A x^{-\alpha}$ as $x \rightarrow \infty$ for $A \in \mathbb{R}$ and $0<\alpha<1$; neither positivity nor regularity assumptions on $u_{0}$ are necessary. Higher-order terms of the asymptotic expansion, as $t \rightarrow \infty$, of solutions are derived. This permits getting a better description of the influence of the behavior of $u_{0}(x)$ as $x \rightarrow \infty$ on the interaction of dissipation with convection in (1.4). The method in [12] differs from that used in [4,2] and bases on an integral equation (the so-called variation of constants or Duhamel formula) satisfied by solutions to (1.2)-(1.4). A similar approach was employed previously in [11], where conservation laws with diffusion and dispersion were considered.

There are also several papers where the long-time behavior of solutions to the Cauchy problem for convection-diffusion equations on the whole space $\mathbb{R}^{n}$ has been studied. For a recent account of this theory we refer the reader to the papers of Escobedo, Vázquez and Zuazua [5, 6, 7]. A treatment of asymptotic properties of solutions of other equations with diffusion, convection, and absorption can be found in $[3,4]$ and in the references therein.

The case of the problem (1.1)-(1.3) is quite different compared to (1.2)(1.4). In particular, since the nonlinear terms have opposite signs, the struc- 
ture of the set of self-similar solutions is completely different in the two cases.

This paper is organized as follows. In the next section, we state and discuss the main results. Section 3 gathers properties of self-similar solutions to the problem (1.1)-(1.3). In Section 4, we study the large-time behavior of solutions to (1.1)-(1.3) with initial data satisfying $\lim _{x \rightarrow \infty} x^{\alpha} u_{0}(x)=A$ for some $A>0$ and $0<\alpha<1$.

Notation. For $1 \leq p \leq \infty$ the $L^{p}\left(\mathbb{R}^{+}\right)$-norm of a Lebesgue measurable real-valued function defined on $\mathbb{R}^{+}=[0, \infty)$ is denoted by $\|f\|_{p}$. The letter $C$ will denote generic positive constants, which do not depend on $t$ and may vary from line to line during computations.

2. Main results. By standard results, the initial-boundary value problem (1.1)-(1.3) has a classical solution $u(x, t)$ which exists locally in $t$ provided that $u_{0}$ is a bounded continuous function. The proof is based on an approximation argument, and it will be omitted. This solution exists, in fact, for all times since, by the well-known comparison principle (cf. e.g. [10]), constant functions are sub- and/or supersolutions to (1.1)-(1.3). Hence, $u(\cdot, t)$ is a bounded function for every $t>0$ if so is $u_{0}$.

Theorem 2.1. Let $q>2$ and $\gamma=1 /(2(q-1))$. There exist positive solutions to (1.1)-(1.2) of special (self-similar) form $u(x, t)=t^{-\gamma} v(x / \sqrt{t})$. Their asymptotic behavior is either

$$
v(\xi)=A e^{-\xi^{2} / 4} \xi^{2 \gamma-1}\left\{1-(2 \gamma-1)(2 \gamma-2) \xi^{-2}+o\left(\xi^{-2}\right)\right\} \quad \text { as } \xi \rightarrow \infty,
$$

or

$$
v(\xi)=A \xi^{-2 \gamma}\left\{1+2 \gamma(2 \gamma+1) \xi^{-2}+o\left(\xi^{-2}\right)\right\} \quad \text { as } \xi \rightarrow \infty
$$

with some positive constant $A$.

REMARK 2.1. Related results have recently been obtained by M. Guedda $[8]$.

Remark 2.2. Figure 1 (plotted using Mathematica) illustrates the behavior of self-similar solutions for $q=3$ and different initial values $v(0)$. In particular, $v(0) \approx 0.672$ corresponds to a solution of the type (2.1) (solid line). Solutions of the type (2.2) are represented by the dashed lines. Note that $\lim _{\xi \rightarrow \infty} \xi^{2 \gamma} v(\xi)$ increases when $v(0) \ll 1$ decreases to 0 . The solution discussed in Remark 3.1 below is drawn with the dotted line.

Our next result concerns general solutions to the initial-boundary value problem (1.1)-(1.3) and their convergence toward the self-similar profiles considered in Theorem 2.1. From now on, we limit ourselves to self-similar 


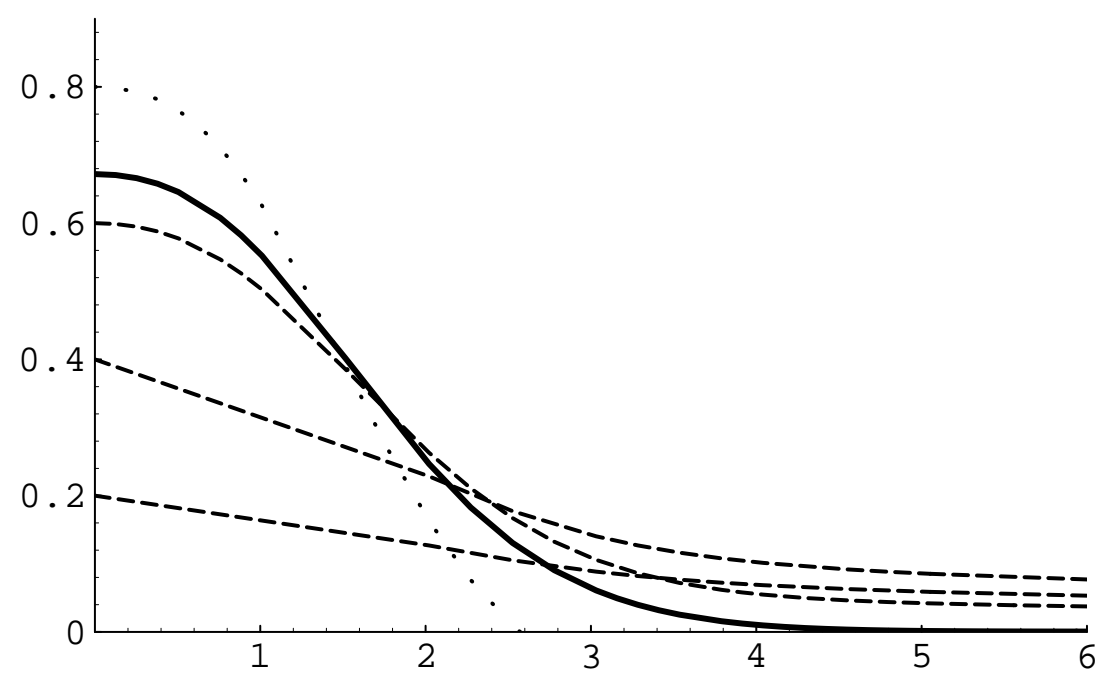

Fig. 1

solutions with the algebraic decay $(2.2)$ as $\xi \rightarrow \infty$. They will be denoted by

$$
u_{A}(x, t) \equiv t^{-\gamma} v_{A}(x / \sqrt{t}),
$$

where $\gamma=1 /(2(q-1))$. In other words, the profile $v_{A}$ is the solution to (3.2)-(3.4) below satisfying the condition

$$
\lim _{\xi \rightarrow \infty} \xi^{2 \gamma} v_{A}(\xi)=A .
$$

We impose the following assumptions on the initial data:

(2.5) there exists a self-similar profile $v_{A}$ and a nonnegative constant $a$ such that $0 \leq u_{0}(x) \leq a^{-\gamma} v_{A}(x / \sqrt{a})$ for all $x \geq 0$, $\lim _{x \rightarrow \infty} x^{2 \gamma} u_{0}(x)=A$.

REMARK 2.3. The asymptotic expansion (2.2) guarantees that every bounded initial data satisfying $0 \leq u_{0}(x) \leq A x^{-2 \gamma}\left\{1+\kappa x^{-2}\right\}$ with $\kappa<$ $2 \gamma(2 \gamma+1)$ also satisfies condition (2.5).

Now we can formulate the theorem.

Theorem 2.2. Suppose that $u_{0}$ and $v_{A}$ satisfy assumptions (2.5) and (2.6). Let $u$ be the solution to (1.1)-(1.3). Then

$$
t^{\gamma}\left\|u(\cdot, t)-u_{A}(\cdot, t)\right\|_{\infty} \rightarrow 0 \quad \text { as } t \rightarrow \infty
$$

provided $v_{A}(0)<\{q \Gamma(1 / 2-\gamma) / \Gamma(1-\gamma)\}^{-1 /(q-1)}$.

REMARK 2.4. For $q=3$ we have $\{q \Gamma(1 / 2-\gamma) / \Gamma(1-\gamma)\}^{-1 /(q-1)} \approx$ 0.335653 . 
3. Self-similar solutions. It is easy to see that the problem (1.1)(1.3) does not have nontrivial traveling wave solutions. Indeed, if $u(x, t)=$ $v(x+c t) \equiv v(\xi)$, then $c v^{\prime}-v^{\prime \prime}+\left(v^{q}\right)^{\prime}=0$, so after integration we get $v^{\prime}(\xi)=$ $v(\xi)^{q}+c v(\xi)-\left(v(0)^{q}-c v(0)\right)$. Taking into account the initial conditions $v^{\prime}(0)=0$ and $v(0) \geq 0$, we obtain $v(\xi) \equiv v(0)$ by the uniqueness of solutions to that initial value problem for a second order ordinary differential equation.

Another class of special solutions to (1.1)-(1.3) consists of self-similar ones, namely those of the form

$$
u(x, t)=t^{-\gamma} v(x / \sqrt{t})
$$

obtained from the invariance properties of (1.1). Here $\gamma=1 /(2(q-1))$, so the function $v$ satisfies

$$
\begin{aligned}
v^{\prime \prime}+\frac{\xi}{2} v^{\prime}+\gamma v-\left(v^{q}\right)^{\prime} & =0, \\
v^{\prime}(0) & =0, \\
v(\xi) & >0,
\end{aligned}
$$

with $\xi=x / \sqrt{t}$ and ${ }^{\prime}=d / d \xi$. As we will see further on, the integrability condition $\int_{0}^{\infty} u(x, t) d x<\infty$, equivalent to $\int_{0}^{\infty} v(\xi) d \xi<\infty$, can be expressed as the requirement

$$
\xi^{2 \gamma} v(\xi) \rightarrow 0 \quad \text { as } \xi \rightarrow \infty .
$$

Note that for $v$ not satisfying the above condition the initial data $u(x, 0)$ does not make sense as the limit of (3.1) as $t \searrow 0$.

Since $0 \leq \int_{0}^{\infty} u(x, t) d x=t^{1 / 2-\gamma} \int_{0}^{\infty} v(\xi) d \xi$, for $\gamma>1 / 2$ we would have a "very singular" solution (in the sense of [1]). But, after integration of (3.2) over $[0, \xi]$, we obtain

$$
v^{\prime}(\xi)+\frac{\xi}{2} v(\xi)+(\gamma-1 / 2) \int_{0}^{\xi} v(\eta) d \eta=v(\xi)^{q}-v(0)^{q},
$$

which leads to

$$
v^{\prime}(\xi) \leq v^{\prime}(\xi)+\frac{\xi}{2} v(\xi)=(1 / 2-\gamma) \int_{0}^{\xi} v(\eta) d \eta+v(\xi)^{q}-v(0)^{q},
$$

so in the limit $\xi \rightarrow \infty$ we arrive at $\gamma<1 / 2$, i.e. $q>2$. This is a consequence of the property

$$
v(\xi), v^{\prime}(\xi) \rightarrow 0 \quad \text { as } \xi \rightarrow \infty
$$

valid for all globally positive solutions to (3.2)-(3.3). A similar property for the equation (3.2) with the reverse $(+)$ sign of the nonlinearity was proved in [13, Lemma 3.1]. Here it follows from the reasoning involving another 
(phase plane) formulation of (3.2):

$$
\begin{aligned}
v^{\prime} & =w, \\
w^{\prime} & =-\frac{\xi}{2} w-\gamma v+q v^{q-1} w .
\end{aligned}
$$

Namely, if $v(\xi)>0$ for all $\xi$, then $v(\xi) \leq v(0)$ and

$$
w^{\prime} \geq-\gamma v+q v^{q-1} w \geq-\gamma v(0)+q v(0)^{q-1} w .
$$

After integration, we get

$$
w(\xi) \geq \exp \left(q v(0)^{q-1} \xi\right)\left(w(0)-\gamma v(0) \int_{0}^{\xi} \exp \left(-q v(0)^{q-1} \eta\right) d \eta\right),
$$

so $w(\xi) \geq-\gamma v(0) C \exp \left(q v(0)^{q-1} \xi\right)$ with $C=\left(q v(0)^{q-1}\right)^{-1}$. For $v>0$ we have

$$
\begin{aligned}
v^{\prime}(\xi) & \leq v^{\prime}(\xi)+\xi v(\xi) / 2=(1 / 2-\gamma) \int_{0}^{\xi} v(\eta) d \eta+v(\xi)^{q}-v(0)^{q} \\
& \leq(1 / 2-\gamma) \int_{0}^{\xi} v(\eta) d \eta .
\end{aligned}
$$

Since $v$ decreases to a limit $\lim _{\xi \rightarrow \infty} v(\xi)=v_{1} \geq 0, v_{1}$ must be 0 -otherwise $v^{\prime}(\xi) \leq-C<0$ for all $\xi \geq 1$, which contradicts the positivity of $v$.

We will prove Theorem 2.1 on the existence of a solution to the problem (3.2)-(3.5) using the shooting method. First, we need a universal a priori upper bound for solutions of (3.2)-(3.4).

LEMMA 3.1. Each positive solution $v$ of (3.2)-(3.3) satisfies

$$
v(\xi) \leq \min \left(c_{\gamma} v(0) \xi^{-2 \gamma}, v(0)\right),
$$

where $c_{\gamma}=1+2 \gamma \int_{0}^{\infty} \eta^{2 \gamma-1} \exp \left(-\eta^{2} / 4\right) d \eta<\infty$.

Proof. (3.2) implies that $\left(v^{\prime}(\xi) e^{\xi^{2} / 4}\right)^{\prime}+\gamma e^{\xi^{2} / 4} v(\xi)=e^{\xi^{2} / 4}\left(v^{q}\right)^{\prime}(\xi) \leq 0$ because positive solutions decrease. Then

$$
e^{\xi^{2} / 4} v^{\prime}(\xi)+\gamma \int_{0}^{\xi} e^{\eta^{2} / 4} v(\eta) d \eta \leq 0
$$

and again by the monotonicity of $v$ we obtain

$$
v^{\prime}(\xi)+\gamma v(\xi) e^{-\xi^{2} / 4} \int_{0}^{\xi} e^{\eta^{2} / 4} d \eta \leq 0 .
$$

Since the elementary inequality 


$$
\frac{\xi}{2} \int_{0}^{\xi} e^{\eta^{2} / 4} d \eta \geq e^{\xi^{2} / 4}-1
$$

holds for all $\xi \geq 0$, we arrive at $v^{\prime}(\xi)+2 \gamma v(\xi) / \xi \leq 2 \gamma v(\xi) e^{-\xi^{2} / 4} / \xi$. After integration we have

$$
v(\xi) \leq \xi^{-2 \gamma}\left(v(0)+2 \gamma v(0) \int_{0}^{\xi} \eta^{2 \gamma-1} e^{-\eta^{2} / 4} d \eta\right),
$$

which implies the conclusion (3.10).

REMARK 3.1. Our next observation is that solutions to (3.2)-(3.4) should have moderate initial conditions $v(0)$. Indeed, from (3.2) we have

$$
\begin{aligned}
v^{\prime}(\xi)+(\gamma-1 / 2) \int_{0}^{\xi} v(\eta) d \eta & \leq v^{\prime}(\xi)+\xi v(\xi) / 2+(\gamma-1 / 2) \int_{0}^{\xi} v(\eta) d \eta \\
& =v(\xi)^{q}-v(0)^{q}
\end{aligned}
$$

and

$$
v(\xi) \leq v(0)+(1 / 2-\gamma) \int_{0}^{\xi} \int_{0}^{\eta} v(\zeta) d \zeta d \eta+\int_{0}^{\xi} v(\eta)^{q} d \eta-\xi v(0)^{q} .
$$

Using the result of Lemma 3.1 we get $v(\xi) \leq v(0)+(1 / 2-\gamma) \xi^{2} v(0)+$ $C v(0)^{q}-\xi v(0)^{q}$, where

$$
\begin{aligned}
\int_{0}^{\infty} v(\eta)^{q} d \eta & \leq \int_{0}^{1} v(0)^{q} d \eta+\int_{1}^{\infty} v(0)^{q} c_{\gamma}^{q} \eta^{-2 \gamma q} d \eta \\
& =v(0)^{q}\left(1+c_{\gamma}^{q} \int_{1}^{\infty} \eta^{-q /(q-1)} d \eta\right) \equiv C v(0)^{q}<\infty
\end{aligned}
$$

Now, given $\xi>C$ there exists $v(0)>0$ such that $v(\xi)<0$ (remember that $q>2>1, \gamma<1 / 2)$, so large initial conditions $v(0)$ cannot lead to globally positive solutions to (3.2)-(3.3).

The goal of the next lemma is twofold: it gives either a (not optimal) lower bound for solutions to (3.2)-(3.4) (not satisfying (3.5)), or an exponential upper bound for a solution to (3.2)-(3.5).

LEMma 3.2. If $v$ is a solution to (3.2)-(3.4), then either

$$
\liminf _{\xi \rightarrow \infty} \xi v(\xi)>0
$$

or

$$
\limsup _{\xi \rightarrow \infty} \exp \left(\alpha \xi^{2} / 2\right) v(\xi)<\infty
$$

for each $\alpha \in[\gamma, 1 / 2)$. 
Pr o of. Define $z(\xi)=v^{\prime}(\xi)+\alpha \xi v(\xi)$ with $\alpha \in \mathbb{R}$. The function $z$ satisfies

$$
z^{\prime}(\xi)=\left(q v(\xi)^{q-1}-(1 / 2-\alpha) \xi\right) v^{\prime}(\xi)+(\alpha-\gamma) v(\xi) .
$$

Thus, for $\alpha \in[\gamma, 1 / 2)$ and all $\xi \geq \xi_{0}=q v(0)^{q-1} /(1 / 2-\alpha)$ we have $z^{\prime}(\xi) \geq 0$, that is, $v^{\prime}(\xi)+\alpha \xi v(\xi)$ increases. Now, two possibilities occur: either there exists a $\xi_{1} \geq \xi_{0}$ such that $z\left(\xi_{1}\right)=\varepsilon>0$, or $z(\xi) \leq 0$ for all $\xi \geq \xi_{0}$. The former implies the inequalities $\varepsilon \leq z(\xi) \leq \alpha \xi v(\xi)$, so $v(\xi) \geq \varepsilon /(\alpha \xi)$ and (3.12) is shown. The latter means $\left(e^{\alpha \xi^{2} / 2} v(\xi)\right)^{\prime} \leq 0$ for $\xi \geq \xi_{0}$, and

$$
v(\xi) \leq e^{-\alpha \xi^{2} / 2} v\left(\xi_{0}\right) e^{\alpha \xi_{0}^{2} / 2}=C(v(0), q) e^{-\alpha \xi^{2} / 2},
$$

which leads to (3.13).

Having the preliminary estimates (3.10), (3.12), (3.13) we can prove the existence of a nontrivial solution of (3.2)-(3.4). Then we will establish the existence of a solution to (3.2)-(3.5), together with the asymptotics as $\xi \rightarrow$ $\infty$ of these solutions.

Lemma 3.3. There exists a solution $v$ to the problem (3.2)-(3.4).

Pr o of. Consider $F(\xi)=v^{\prime}(\xi) / v(\xi)$ which is well defined for small $\xi>0$ as $v(0)>0$. From equation (3.2) we obtain $F^{\prime}=-\xi F / 2-\gamma+q v^{q-1} F-F^{2} \geq$ $-\gamma+q v(0)^{q-1} F-F^{2}$ because $v^{\prime} \leq 0$ as long as $v>0$ exists. Putting $c=$ $q v(0)^{q-1}$, which is small together with the initial condition $v(0)$, we rewrite the preceding relation as $F^{\prime}\left(F^{2}-c F+\gamma\right)^{-1} \geq-1$. After an elementary integration we get

$$
F(\xi) \geq-\left(\gamma-c^{2} / 4\right)^{1 / 2} \tan \left(\left(\gamma-c^{2} / 4\right) \xi+\arctan \left(\frac{c}{2\left(\gamma-c^{2} / 4\right)^{1 / 2}}\right)\right)
$$

If $\xi_{0}=1 /(2 \gamma)$ (this is possible for $c$ small enough), then for all $\xi \in\left[0, \xi_{0}\right]$ we have $F(\xi)>-\infty\left(\right.$ so $v(\xi)$ makes sense), and even $F\left(\xi_{0}\right) \geq-\left(\gamma-c^{2} / 4\right)^{1 / 2} / \sqrt{3}$ (as $\tan 1 / 2<1 / \sqrt{3}$ ).

Now, define the quantity $z(\xi)=v^{\prime}(\xi)+\gamma \xi v(\xi)$ satisfying the relation $z^{\prime}(\xi)=v^{\prime}(\xi)\left(q v(\xi)^{q-1}-(1 / 2-\gamma) \xi\right)$. Clearly, $z^{\prime}(\xi) \geq 0$ for all $\xi \geq \xi_{1} \equiv$ $c /(1 / 2-\gamma)$. Since $\xi_{1}<\xi_{0}$ for small $c>0, v^{\prime}(\xi)+\gamma \xi v(\xi)$ increases starting from a point where the estimate (3.14) is still valid.

Consider $\xi_{2}=\sup \{\xi: F(\xi) \geq-\sqrt{\gamma / 3}\}$, which is positive by (3.3). The case $\xi_{2}=\infty$ is easy: $v(\xi) \geq v(0) \exp (-\sqrt{\gamma / 3} \xi)$ for all $\xi \geq 0$, which together with (3.10) gives the existence of a solution $v$ to (3.2)-(3.4) such that $v(\xi) \rightarrow 0$ as $\xi \rightarrow \infty$.

Otherwise, $\xi_{2}<\infty$ and $z\left(\xi_{2}\right)=v\left(\xi_{2}\right)\left(-\sqrt{\gamma / 3}+\gamma \xi_{2}\right)>0$ because $\xi_{2}>$ $\xi_{0}=1 /(2 \gamma), \gamma<1 / 2$. Then we have $\left(v(\xi) e^{\gamma \xi^{2} / 2}\right)^{\prime} \geq \nu e^{\gamma \xi^{2} / 2}>0$ for all $\xi \geq \xi_{1}$ (hence for all $\xi \geq \xi_{2}$ ) and $\nu \equiv \gamma \xi_{2}-\sqrt{\gamma / 3}>0$. The integration from 
$\xi_{2}$ to $\xi$ leads to

$$
v(\xi) \geq e^{-\gamma \xi^{2} / 2+\gamma \xi_{2}^{2} / 2} v\left(\xi_{2}\right)+\nu e^{-\gamma \xi^{2} / 2} \int_{\xi_{2}}^{\xi} e^{\gamma \eta^{2} / 2} d \eta
$$

and (applying a modification of inequality (3.11)) $v(\xi) \geq C e^{-\gamma \xi^{2} / 2} v\left(\xi_{2}\right)+$ $2 \nu /(\gamma \xi)$. Such a solution starting at a small $v(0)$ exists for all $\xi \geq 0$ but it does not satisfy the condition (3.5).

The further analysis of self-similar solutions follows, roughly speaking, the lines of the analysis of solutions to a related problem in [1]. Since our solutions must have small initial data $v(0) \geq v(\xi)$, we expect that their asymptotics will be determined by that of the linearized equation

$$
v^{\prime \prime}(\xi)+\frac{\xi}{2} v^{\prime}(\xi)+\gamma v(\xi)=0 .
$$

Recall from [1] that (3.15) has solutions $v_{1}, v_{2}$ with different asymptotic behavior at $\infty$ :

$$
\begin{aligned}
& v_{1}(\xi)=A e^{-\xi^{2} / 4} \xi^{2 \gamma-1}\left\{1-(2 \gamma-1)(2 \gamma-2) \xi^{-2}+o\left(\xi^{-2}\right)\right\}, \\
& v_{2}(\xi)=A \xi^{-2 \gamma}\left\{1+2 \gamma(2 \gamma+1) \xi^{-2}+o\left(\xi^{-2}\right)\right\}
\end{aligned}
$$

as $\xi \rightarrow \infty$. We will prove that there is a solution to (3.2)-(3.5) (the socalled fast solution of the system (3.8)-(3.9)) and its asymptotic behavior is determined by $v_{1}$ with a suitable $A>0$ (as in (2.1)), while solutions to (3.2)-(3.4) (the so-called slow solutions of (3.8)-(3.9)) decay like $v_{2}$ (cf. $(2.2))$.

LEMmA 3.4. The sector $L_{\lambda}=\{w \geq-\lambda v\}, \lambda>0$, is invariant for the system (3.8)-(3.9) if $\xi \geq \xi_{\lambda}$ is sufficiently large.

P r o of (cf. [1, Lemma 2]). It suffices to check that on the line $w=-\lambda v$ we have $w^{\prime} / v^{\prime}=-\xi / 2-\gamma v / w+q v^{q-1}<-\lambda$ for $\xi \geq \xi_{\lambda}=2(\lambda+\gamma / \lambda+$ $\left.q v(0)^{q-1}\right)$.

Lemma 3.5. For each trajectory of (3.8)-(3.9) with positive $v$, the limit $\ell=\lim _{\xi \rightarrow \infty} w(\xi) / v(\xi)$ exists and is either 0 or $-\infty$.

P r o of (cf. [1, Lemmata 5-7]). From the invariance properties of sectors $L_{\lambda}$ the existence of the limit $\ell \in[-\infty, 0]$ is clear. Its actual value can be calculated using the de l'Hôpital rule:

$$
\begin{aligned}
\ell & =\lim _{\xi \rightarrow \infty} v^{\prime} / v=\lim _{\xi \rightarrow \infty} w^{\prime} / w=\lim _{\xi \rightarrow \infty}\left(q v^{q-1}-\gamma v / w-\xi / 2\right) \\
& =\lim _{\xi \rightarrow \infty}(-\gamma v / w-\xi / 2) \\
& =\lim _{\xi \rightarrow \infty}\left(-\xi / 2+v^{q-1}+v^{-1}\left((1 / 2-\gamma) \int_{0}^{\xi} v-v(0)^{q}\right)\right)
\end{aligned}
$$




$$
=\lim _{\xi \rightarrow \infty}\left(-\xi / 2+v^{-1}\left((1 / 2-\gamma) \int_{0}^{\xi} v-v(0)^{q}\right)\right),
$$

which implies that $-\infty-\gamma / \ell=\ell$, so either $\ell=0^{-}$or $\ell=-\infty$.

Thus, we have two possibilities: either $w / v$ increases to 0 as $\xi \rightarrow \infty$ (like a slow solution), or $w / v$ decreases to $-\infty$ (like a fast solution). We know from Lemma 3.3 that the set

$$
S=\left\{v(0): \lim _{\xi \rightarrow \infty} v(\xi)=0, \lim _{\xi \rightarrow \infty} v^{\prime}(\xi) / v(\xi)=0\right\}
$$

of initial values of slow solutions is not empty (moreover, $\mathbb{R}^{+} \backslash S \neq \emptyset$ by the observation after Lemma 3.1). $S$ is an open set (cf. [1, Lemma 9]). The solution corresponding to $v(0)=v^{*} \equiv \sup S$ is of particular interest. We prove that this is a fast solution to (3.8)-(3.9). We do not know if this fast solution is unique.

LEMma 3.6. The function $v^{*}(\xi)=\lim _{k \rightarrow \infty} v_{k}(\xi)$, where $v_{k}$ satisfies (3.2)-(3.3) and $v_{k}(0)=v_{k}$ with $v_{k} \nearrow v^{*}=\sup S$, is a solution of (3.2)-(3.4) and $v^{*}(\xi)=\mathcal{O}\left(e^{-\lambda \xi}\right)$ for all $\lambda>0$.

Proof (cf. [1, Lemma 11]). Clearly, $v^{*}(\xi) \geq 0$ for each $\xi \geq 0$ by the continuous dependence on initial data. Suppose that $v^{*}\left(\xi_{0}\right)=0$ for some $\xi_{0}>0$. Then $\left(v^{*}\right)^{\prime}\left(\xi_{0}\right)<0$ by the uniqueness of solutions to second order equations, $v^{*}(\xi)<0$ for $\xi_{0}<\xi<\xi_{0}+\delta$ with some $\delta>0$, which contradicts the positivity of $v^{*}$. By Lemma 3.5, $\ell=-\infty$ ( $v^{*} \notin S$ because $S$ is open). Thus for each $\lambda>0$ and all sufficiently large $\xi$ we have $v^{\prime}(\xi) / v(\xi) \leq-\lambda$, which implies the exponential decay of $v^{*}$.

The asymptotic behavior as $\xi \rightarrow \infty$ of $v^{*}$ is obtained by a repeated use of the de l'Hôpital rule.

Lemma 3.7. There exists a constant $A>0$ such that

$$
v^{*}(\xi)=A e^{-\xi^{2} / 4} \xi^{2 \gamma-1}\left\{1-(2 \gamma-1)(2 \gamma-2) \xi^{-2}+o\left(\xi^{-2}\right)\right\}
$$

as $\xi \rightarrow \infty$.

Proof (cf. [1, Lemmata 13-15]). First we show $\lim _{\xi \rightarrow \infty} w(\xi) /(\xi v(\xi))$ $=-1 / 2$ for $v \equiv v^{*}$. Indeed,

$$
\begin{aligned}
\lim _{\xi \rightarrow \infty} w(\xi) /(\xi v(\xi)) \\
\quad=\lim _{\xi \rightarrow \infty} w^{\prime}(\xi) /\left(\xi v^{\prime}(\xi)+v(\xi)\right) \\
\quad=\lim _{\xi \rightarrow \infty}\left(-1 / 2-\gamma v(\xi) /(\xi w(\xi))+q v(\xi)^{q-1} / \xi\right)(1+v(\xi) /(\xi w(\xi)))^{-1} \\
\quad=-1 / 2 .
\end{aligned}
$$


Next consider $E(\xi)=\xi v^{\prime}(\xi)+\frac{1}{2} \xi^{2} v(\xi)$. A similar calculation gives

$$
\begin{aligned}
\lim _{\xi \rightarrow \infty} & E(\xi) / v(\xi) \\
& =\lim _{\xi \rightarrow \infty} E^{\prime}(\xi) / v^{\prime}(\xi)=\lim _{\xi \rightarrow \infty}\left(1+\xi v^{\prime \prime}(\xi) / v^{\prime}(\xi)+\xi v(\xi) / v^{\prime}(\xi)+\xi^{2}\right) \\
& =\lim _{\xi \rightarrow \infty}\left(1+\xi(1-\gamma) v(\xi) / v^{\prime}(\xi)\right)=1+(1-\gamma)(-2)=2 \gamma-1 .
\end{aligned}
$$

The last auxiliary quantity is $G(\xi)=\xi^{2} E(\xi)+(1-2 \gamma) \xi^{2} v(\xi)$. We compute $\lim _{\xi \rightarrow \infty} G(\xi) / v(\xi)=(1-\gamma)(1-2 \gamma)$. Again from the de l'Hôpital rule, we need to compute

$$
\begin{aligned}
\xi^{2} & E^{\prime}(\xi) / v^{\prime}(\xi) \\
& =\xi^{2}\left(1+(1-\gamma) \xi v(\xi) / v^{\prime}(\xi)+\xi q v(\xi)^{q-1}\right) \\
& =\xi^{2}\left(1+(1-\gamma)\left(\xi v(\xi) / v^{\prime}(\xi)+2\right)-2(1-\gamma)\right)+\ldots \\
& =\xi^{2}\left((2 \gamma-1)+(1-\gamma)\left(\xi v(\xi)+2 v^{\prime}(\xi)\right)(\xi v(\xi))^{-1} \xi v(\xi) / v^{\prime}(\xi)\right)+\ldots
\end{aligned}
$$

This information permits us to write

$$
\begin{aligned}
& G(\xi) / v(\xi)=(1-\gamma)(1-2 \gamma)+o(1), \\
& E(\xi) / v(\xi)=(2 \gamma-1)+(1-\gamma)(1-2 \gamma) \xi^{-2}+o(1) \xi^{-2}, \\
& v^{\prime}(\xi) / v(\xi)=(2 \gamma-1) \xi^{-1}-\xi / 2+(1-\gamma)(1-2 \gamma) \xi^{-3}+o(1) \xi^{-3},
\end{aligned}
$$

and after an integration

$$
\log v(\xi) / v(1)=\log \xi^{2 \gamma-1}-\xi^{2} / 4-(1-\gamma)(1-2 \gamma)\left(2 \xi^{2}\right)^{-1}+\int_{1}^{\xi} o(1) \eta^{-3} d \eta,
$$

which is equivalent to the conclusion of Lemma 3.7.

A similar reasoning leads to the asymptotic estimate (2.2) for slow solutions, which ends the proof of Theorem 2.1.

4. Stability of the self-similar profiles. The main goal of this section is to prove Theorem 2.2. However, we begin with recalling briefly some properties of solutions to the auxiliary linear problem

$$
\begin{aligned}
u_{t}-u_{x x} & =0, \quad x>0, t>0, \\
u_{x}(0, t) & =0, \\
u(x, 0) & =u_{0}(x) .
\end{aligned}
$$

The reader is referred to [12, Section 3] for more details about this Neumann problem for the heat equation.

For a function $f$ on $[0, \infty)$ we define the even continuation $\tilde{f}$ to $\mathbb{R}$ by

$$
\widetilde{f}(x)= \begin{cases}f(x) & \text { for } x \geq 0, \\ f(-x) & \text { for } x<0 .\end{cases}
$$


In this notation, for every $u_{0} \in L^{1}\left(\mathbb{R}^{+}\right)+L^{\infty}\left(\mathbb{R}^{+}\right)$, the unique classical solution to the problem (4.1)-(4.3) is given by

$$
\begin{aligned}
e^{t \Delta} u_{0}(x) & \equiv \int_{\mathbb{R}} G(x-y, t) \widetilde{u}_{0}(y) d y \\
& =\int_{\mathbb{R}^{+}}(G(x-y, t)+G(x+y, t)) u_{0}(x) d y,
\end{aligned}
$$

where the function $G(x, t)=(4 \pi t)^{-1 / 2} \exp \left(-x^{2} /(4 t)\right)$ is the Gauss-Weierstrass kernel. Moreover, for the heat semigroup with the homogeneous Neumann condition (4.2) we have

$$
\left(e^{t \Delta} v_{x}\right)(x)=-2 G(x, t) v(0)-\left(e^{t \Delta} v\right)_{x}(x)
$$

for every sufficiently regular $v=v(x)(x \in[0, \infty))$, which is obtained by integrating by parts in (4.4).

We also recall that for $1 \leq r \leq p \leq \infty$ and $f \in L^{r}\left(\mathbb{R}^{+}\right)$there exists a constant $C>0$ independent of $f$ and $t$ such that

$$
\left\|e^{t \Delta} f\right\|_{p} \leq 2\|G(\cdot, t)\|_{p r /(p r+r-p)}\|f\|_{r} \leq C t^{-(1 / r-1 / p) / 2}\|f\|_{r},
$$

and

$$
\left\|\left(e^{t \Delta} f\right)_{x}\right\|_{p} \leq 2\left\|G_{x}(\cdot, t)\right\|_{p r /(p r+r-p)}\|f\|_{r} \leq C t^{-(1 / r-1 / p) / 2-1 / 2}\|f\|_{r}
$$

for all $t>0$. This is an immediate consequence of formula (4.4) and the Young inequality (cf. [12, Lemma 3.3]).

Lemma 4.1. Assume that $u_{0} \in L^{\infty}\left(\mathbb{R}^{+}\right)$satisfies (2.6). For every $p \in$ $(q-1, \infty]$,

$$
t^{\gamma-1 /(2 p)}\left\|e^{t \Delta}\left(u_{0}(\cdot)-A(\cdot)^{-2 \gamma}\right)\right\|_{p} \rightarrow 0 \quad \text { as } t \rightarrow \infty .
$$

Proof. Recall that $2 \gamma<1$ is equivalent to $q>2$. The proof of this lemma is classical and bases on explicit expressions for $e^{t \Delta} u_{0}$ and $e^{t \Delta}(\cdot)^{-2 \gamma}$. Indeed, we have

$$
\left(G(\cdot, t) * \widetilde{u}_{0}\right)(x)=A e^{t \Delta}|x|^{-2 \gamma}(1+o(1)) \quad \text { as } t \rightarrow \infty,
$$

uniformly in $x \in \mathbb{R}$ (cf. e.g. [9, Lemma 3.3.c]). Hence, to complete the proof, we use the fact that $e^{t \Delta}(\cdot)^{-2 \gamma} \in L^{p}\left(\mathbb{R}^{+}\right)$for $p \in(q-1, \infty]$ together with the estimate

$$
\left\|e^{t \Delta}(\cdot)^{-2 \gamma}\right\|_{p}=\left\|e^{\Delta}(\cdot)^{-2 \gamma}\right\|_{p} t^{-\gamma+1 /(2 p)} .
$$

Let us also formulate an auxiliary well-known fact.

Lemma 4.2. Assume that $\left\{f_{i}\right\}_{i>0}$ is a sequence of measurable functions on $[0,1]$ and $h \in L^{1}(0,1)$ is such that for every $i \geq 0$ and $x \in[0,1],\left|f_{i}(x)\right| \leq$ 
$h(x)$. Define $\lim \sup _{i \rightarrow \infty} f_{i}(x) \equiv \lim _{k \in \mathbb{N}, k \rightarrow \infty} \sup _{i>k} f_{i}(x)$. Then

$$
\limsup _{i \rightarrow \infty} \int_{0}^{1} f_{i}(x) d x \leq \int_{0}^{1} \limsup _{i \rightarrow \infty} f_{i}(x) d x
$$

Proof. To see this, it suffices to apply the Lebesgue Dominated Convergence Theorem to the obvious inequality

$$
\sup _{i>k} \int_{0}^{1} f_{i}(x) d x \leq \int_{0}^{1} \sup _{i>k} f_{i}(x) d x .
$$

Now, we come back to the nonlinear problem (1.1)-(1.3). It is well known that each sufficiently regular solution satisfies the integral equation

$$
u(t)=e^{t \Delta} u_{0}-\int_{0}^{t} e^{(t-\tau) \Delta}\left(u^{q}\right)_{x}(\tau) d \tau .
$$

Moreover, integrating by parts in the second term on the right-hand side of (4.9) (i.e. using formula (4.5) above), we obtain the equivalent equation

$$
u(x, t)=e^{t \Delta} u_{0}(x)+2 \int_{0}^{t} G(x, t-\tau) u(0, \tau)^{q} d \tau+\int_{0}^{t}\left(e^{(t-\tau) \Delta} u^{q}\right)_{x}(x, \tau) d \tau
$$

This formula is the main tool in the proof of Theorem 2.2. Note that it also holds true when $u$ and $u_{0}$ are replaced by $u_{A}$ and $A x^{-2 \gamma}$, respectively.

Proposition 4.1. Suppose that $u_{0}$ and $v_{A}$ satisfy assumption (2.5). Let $u$ be the solution to (1.1)-(1.3). Then

$$
0 \leq u(x, t) \leq(t+a)^{-\gamma} v_{A}(x / \sqrt{t+a}) \quad \text { for all } t \geq 0 \text { and } x \geq 0 .
$$

P r o of. This follows from the standard comparison principle (cf. [10]).

Since $v_{A}(\xi)$ is decreasing for $\xi>0$ (cf. (3.7)) we immediately obtain the $L^{\infty}$-bound for solutions to (1.1)-(1.3).

Corollary 4.1. Under the assumptions of Proposition 4.1,

$$
\|u(\cdot, t)\|_{\infty} \leq(t+a)^{-\gamma} v_{A}(0) \quad \text { for all } t \geq 0 .
$$

Proposition 4.2. Suppose that $u_{0}$ and $v_{A}$ satisfy assumptions (2.5) and (2.6). Let $u$ be the solution to (1.1)-(1.3). Then $\lim _{t \rightarrow \infty} t^{\gamma} u(0, t)=v_{A}(0)$ provided $v_{A}(0)<\{q \Gamma(1 / 2-\gamma) / \Gamma(1-\gamma)\}^{-1 /(q-1)}$.

Proof. Substituting $x=0$ in (4.5) gives $\left(e^{t \Delta} v_{x}\right)(0)=-(\pi t)^{-1 / 2} v(0)$, because $\left(e^{t \Delta} v\right)_{x}(0)=0$ by (4.4). Hence, for $x=0$ formula (4.10) has the following form:

$$
u(0, t)=2 \int_{\mathbb{R}^{+}} G(y, t) u_{0}(y) d y+\pi^{-1 / 2} \int_{0}^{t}(t-\tau)^{-1 / 2} u(0, \tau)^{q} d \tau .
$$


Now, we subtract this equation from an analogous expression for $u_{A}$ to obtain

$$
\begin{aligned}
u_{A}(0, t)-u(0, t)= & 2 \int_{\mathbb{R}^{+}} G(y, t)\left(A y^{-2 \gamma}-u_{0}(y)\right) d y \\
& +\pi^{-1 / 2} \int_{0}^{t}(t-\tau)^{-1 / 2}\left(u_{A}(0, \tau)^{q}-u(0, \tau)^{q}\right) d \tau .
\end{aligned}
$$

Now note that by Corollary 4.1 we have

$$
0 \leq u(0, t) \leq(t+a)^{-\gamma} v_{A}(0) \leq t^{-\gamma} v_{A}(0)
$$

for all $t>0$. Moreover, since the elementary inequality

$$
\left|X^{q}-Y^{q}\right| \leq \frac{q}{2}|X-Y|\left(X^{q-1}+Y^{q-1}\right)
$$

holds for all nonnegative $X, Y$ and $q \geq 2$ (this is an easy consequence of the fact that the function $\frac{q}{2}(1-t)\left(1+t^{q-1}\right)+t^{q}-1$ is decreasing on $\left.[0,1]\right)$, we obtain immediately

$$
\left|u_{A}(0, \tau)^{q}-u(0, \tau)^{q}\right| \leq q \tau^{-1 / 2} v_{A}(0)^{q-1}\left|u_{A}(0, \tau)-u(0, \tau)\right| .
$$

Hence, multiplying (4.13) by $t^{\gamma}$, using (4.15), and changing the variable $\tau=t s$ we obtain

$$
\begin{aligned}
& t^{\gamma}\left|u_{A}(0, t)-u(0, t)\right| \\
\leq & 2 t^{\gamma} \int_{\mathbb{R}^{+}} G(y, t)\left|A y^{-2 \gamma}-u_{0}(y)\right| d y \\
& +q \pi^{-1 / 2} v_{A}(0)^{q-1} \int_{0}^{1}(1-s)^{-1 / 2} s^{-1 / 2-\gamma}(t s)^{\gamma}\left|v_{A}(0, \tau)-u(0, \tau)\right| d \tau .
\end{aligned}
$$

The first term on the right-hand side of (4.16) tends to 0 as $t \rightarrow \infty$, because this is a particular case of Lemma 4.1. Hence, applying Lemma 4.2 to (4.16) we obtain

$$
\begin{aligned}
& \limsup _{t \rightarrow \infty} t^{\gamma}\left|u_{A}(0, t)-u(0, t)\right| \\
& \quad \leq q \pi^{-1 / 2} v_{A}(0)^{q-1} \int_{0}^{1}(1-s)^{-1 / 2} s^{-1 / 2-\gamma} d s \limsup _{t \rightarrow \infty} t^{\gamma}\left|u_{A}(0, t)-u(0, t)\right| .
\end{aligned}
$$

But $\int_{0}^{1}(1-s)^{-1 / 2} s^{-1 / 2-\gamma} d s=\pi^{1 / 2} \Gamma(1 / 2-\gamma) / \Gamma(1-\gamma)$, hence

$$
\lim _{t \rightarrow \infty} t^{\gamma}\left|u_{A}(0, t)-u(0, t)\right|=0
$$

provided $q \Gamma(1 / 2-\gamma) / \Gamma(1-\gamma) v_{A}^{q-1}(0)<1$.

Now we are ready to prove Theorem 2.2. 
Proof of Theorem 2.2. The reasoning here is similar to that in the proof of Proposition 4.2. We begin with the identity

$$
\begin{aligned}
u(x, t)-u_{A}(x, t)= & e^{t \Delta}\left(u_{0}(x)-A x^{-2 \gamma}\right) \\
& +2 \int_{0}^{t} G(x, t-\tau)\left(u^{q}-u_{A}^{q}\right)(0, \tau) d \tau \\
& +\int_{0}^{t}\left(e^{(t-\tau) \Delta}\left(u^{q}-u_{A}^{q}\right)\right)_{x}(x, \tau) d \tau
\end{aligned}
$$

obtained from (4.10) for $u$ and $u_{A}$. Combining, as in the proof of inequality (4.15), Corollary 4.1 with (4.14), we obtain

$$
\left\|u(\cdot, \tau)^{q}-u_{A}(\cdot, \tau)^{q}\right\|_{\infty} \leq q \tau^{-1 / 2} v_{A}(0)^{q-1}\left\|u(\cdot, \tau)-u_{A}(\cdot, \tau)\right\|_{\infty} .
$$

Let us also recall that the explicit expression for the heat kernel gives immediately

$$
2\|G(\cdot, t-\tau)\|_{\infty}=\pi^{-1 / 2}(t-\tau)^{-1 / 2}
$$

and

$$
2\left\|G_{x}(\cdot, t-\tau)\right\|_{1}=2 \pi^{-1 / 2}(t-\tau)^{-1 / 2} \int_{\mathbb{R}^{+}} z e^{-z^{2}} d z=\pi^{-1 / 2}(t-\tau)^{-1 / 2} .
$$

These facts allow us to compute the $L^{\infty}\left(\mathbb{R}^{+}\right)$-norm of (4.17) and to obtain by (4.7) an inequality analogous to (4.16):

$$
\begin{aligned}
& t^{\gamma}\left\|u(\cdot, t)-u_{A}(\cdot, t)\right\|_{\infty} \\
& =t^{\gamma}\left\|e^{t \Delta}\left(u_{0}-A(\cdot)^{-2 \gamma}\right)\right\|_{\infty} \\
& \quad+2 t^{\gamma} \int_{0}^{t}\|G(\cdot, t-\tau)\|_{\infty}\left|\left(u^{q}-u_{A}^{q}\right)(0, \tau)\right| d \tau \\
& \quad+2 t^{\gamma} \int_{0}^{t}\left\|G_{x}(\cdot, t-\tau)\right\|_{1}\left\|\left(u^{q}-u_{A}^{q}\right)(\cdot, \tau)\right\|_{\infty} d \tau \\
& \leq t^{\gamma}\left\|e^{t \Delta}\left(u_{0}-A(\cdot)^{-2 \gamma}\right)\right\|_{\infty} \\
& \quad+\pi^{-1 / 2} \int_{0}^{1}(1-s)^{-1 / 2} s^{-q \gamma}\left((t s)^{q \gamma}\left|\left(u^{q}-u_{A}^{q}\right)(0, t s)\right|\right) d s \\
& \quad+q \pi^{-1 / 2} v_{A}(0)^{q-1} \\
& \quad \times \int_{0}^{1}(1-s)^{-1 / 2} s^{-1 / 2-\gamma}\left((t s)^{\gamma}\left\|u(\cdot, t s)-u_{A}(\cdot, t s)\right\|_{\infty}\right) d s .
\end{aligned}
$$


The first term on the right-hand side of (4.19) tends to 0 as $t \rightarrow \infty$ by Lemma 4.1. The analogous conclusion holds true for the second term, if one combines Proposition 4.2 with the Lebesgue Dominated Convergence Theorem. Hence, by the argument used at the end of the proof of Proposition 4.2 , we conclude that $\lim _{t \rightarrow \infty} t^{\gamma}\left\|u(\cdot, t)-u_{A}(\cdot, t)\right\|_{\infty}=0$ provided $q \Gamma(1 / 2-$ $\gamma) / \Gamma(1-\gamma) v_{A}^{q-1}(0)<1$.

Acknowledgements. Grant support from KBN 324/P03/97/12, POLONIUM 98111, and the University of Wrocław funds 2204/W/IM/98 is gratefully acknowledged. The authors thank M. Guedda from Université de Picardie (Amiens) for interesting discussions on an alternative proof of Lemma 3.3 during his stay in Wrocław in December 1998, and in February 1999 while P. B. was visiting Amiens. G. K. acknowledges the support from the Foundation for Polish Science.

\section{References}

[1] H. Brezis, L. A. Peletier and D. Terman, A very singular solution of the heat equation with absorption, Arch. Rational Mech. Anal. 95 (1986), 185-209.

[2] S. Claudi, Asymptotic behaviour for a diffusion-convection equation with rapidly decreasing initial data, Adv. Differential Equations 3 (1998), 361-386.

[3] S. Claudi and F. R. Guarguaglini, Large time behavior for the heat equation with absorption and convection, Adv. Appl. Math. 16 (1995), 377-401.

[4] S. Claudi, R. Natalini and A. Tesei, Large time behaviour of a diffusion equation with strong convection, Ann. Scuola Norm. Sup. Pisa Cl. Sci. (4) 3 (1994), 445-474.

[5] M. Escobedo, J. L. Vázquez and E. Zuazua, Asymptotic behaviour and source-type solutions for a diffusion-convection equation, Arch. Rational Mech. Anal. 124 (1993), 43-65.

[6] - - - - - A diffusion-convection equation in several space dimensions, Indiana Univ. Math. J. 42 (1993), 1413-1440.

[7] M. Escobedo and E. Zuazua, Large time behavior for convection-diffusion equations in $\mathbb{R}^{N}$, J. Funct. Anal. 100 (1991), 119-161.

[8] M. Guedda, Self-similar solutions to a convection-diffusion process, Electron. J. Qual. Theory Differ. Equ. 2000, no. 3, 17 pp.

[9] L. Herraiz, Asymptotic behaviour of solutions of some semilinear parabolic problems, Ann. Inst. H. Poincaré Anal. Non Linéaire 16 (1999), 49-105.

[10] S. Kaplan, On the growth of solutions of quasi-linear parabolic equations, Comm. Pure Appl. Math. 16 (1963), 305-330.

[11] G. Karch, Large-time behavior of solutions to nonlinear wave equations: higherorder asymptotics, Math. Methods Appl. Sci. 22 (1999), 1671-1697.

[12] - Asymptotics of solutions to a convection-diffusion equation on the half-line, Proc. Roy. Soc. Edinburgh Sect. A 130 (2000), 837-853. 
[13] L. A. Peletier and H. C. Serafini, A very singular solution and other selfsimilar solutions of the heat equation with convection, Nonlinear Anal. 24 (1995), 29-49.

Mathematical Institute

Wrocław University

Pl. Grunwaldzki 2/4

50-384 Wrocław, Poland

E-mail: biler@math.uni.wroc.pl

karch@math.uni.wroc.pl

Reçu par la Rédaction le 18.3.1999 